\title{
Research on Design of Vibration Platform of Molded Case Circuit Breaker and Reliability of Platform Frame
}

\author{
Jiaguo Yao, Ying Pan \\ College of Mechanical Engineering, Shanghai University of Engineering Science, Shanghai, China \\ Email address: \\ 1137874107@qq.com (Jiaguo Yao),ap_xjtu@163.com (Ying Pan)
}

To cite this article:

Jiaguo Yao, Ying Pan. Research on Design of Vibration Platform of Molded Case Circuit Breaker and Reliability of Platform Frame. American Journal of Engineering and Technology Management. Vol. 3, No. 2, 2018, pp. 35-41. doi: 10.11648/j.ajetm.20180302.11

Received: May 8, 2018; Accepted: May 25, 2018; Published: June 13, 2018

\begin{abstract}
With the continuous development and improvement of modern transportation systems, the demand for transport equipment has been increasing day by day, and low-voltage electrical appliances have become an indispensable part of transport equipment. In the railroad highway navigation and transportation, strong vibration, impact and natural mechanical environment have become one of the important factors affecting the stable, reliable and safe operation of electrical equipment. Molded case circuit breakers generally study their performance index under static conditions, and there are few researches on them under dynamic vibration environment. Therefore, the vibration platform test of molded case circuit breakers is established here to fill in the dynamic test of low-voltage electrical apparatus. The blank space. In addition, in order to ensure the stability and longevity of the overall framework of the designed platform, the reliability of the established test platform framework is analyzed on the basis of ansys analysis, and it is concluded that the established platform framework can guarantee the dynamic testing under vibration. The overall stability will not cause vibration damage to the frame.
\end{abstract}

Keywords: Molded Case Circuit Breaker, Vibration Platform, Frame, Ansys Analysis

\section{Introduction}

This paper mainly studies the reliability of circuit breaker under dynamic load, establishes corresponding vibration test system, and analyzes the structural static and dynamic characteristics of the mechanical arm of the three-way stretching mechanism. A mechanical analysis method for the failure of low-voltage circuit breakers under vibration excitation is presented. The mechanical characteristics model of low-voltage circuit breakers is established. The characteristics of the response of low-voltage circuit breakers under vibration excitation are analyzed. This method can be used to obtain the low-voltage circuit breakers subjected to simple harmonics. Under the excitation (sinusoidal vibration) load, the vibration response and characteristics of the internal moving contact are analyzed. The sinusoidal excitation test and the random excitation vibration test were performed for the 3VT8 molded case circuit breaker and the 3WT8 frame circuit breaker.

\section{Design and Implementation of Reliability Test Platform for} Low-Voltage Electrical Apparatus for Variable Load Download

Based on Dongling ES-60WLS3-445 electromagnetic vibrating table and controller, a reliability test hardware platform consisting of a vibratory table system, a three-dimensional sliding positioning mechanism, a robot arm stretching mechanism, a low-voltage electrical apparatus for load, a stress strain gauge, and an oscilloscope was established.. The mechanical properties of the load-carrying appliances were analyzed and the kinematics of the manipulator's stretching mechanism were studied. In order to achieve the reliability test of the critical on-off state of the load-bearing appliance, a reliability test system for the load-carrying appliance was established. [1]

(1) Design a set of $\mathrm{X}, \mathrm{Y}, \mathrm{Z}$ axis three-dimensional sliding table positioning mechanism

This technology is aimed at the stability test requirements 
of the load-carrying appliances. The $\mathrm{X}, \mathrm{Y}$, and Z-axis three-dimensional sliding table positioning mechanisms are designed so that the traction mechanism can reach any point in space within a certain range. Moreover, in order to ensure that the three-dimensional sliding platform can test the stability of load-carrying appliances in a vibrating environment, a screw and a latch mechanism are jointly fixed to fix the design of the three-dimensional sliding platform. At the same time, according to the requirements of the traction action of the moving and static contact, a rigid actuator with a hooking method and a Y-axis linkage was designed to improve the accuracy and anti-jamming ability of the traction mechanism under the vibration environment.

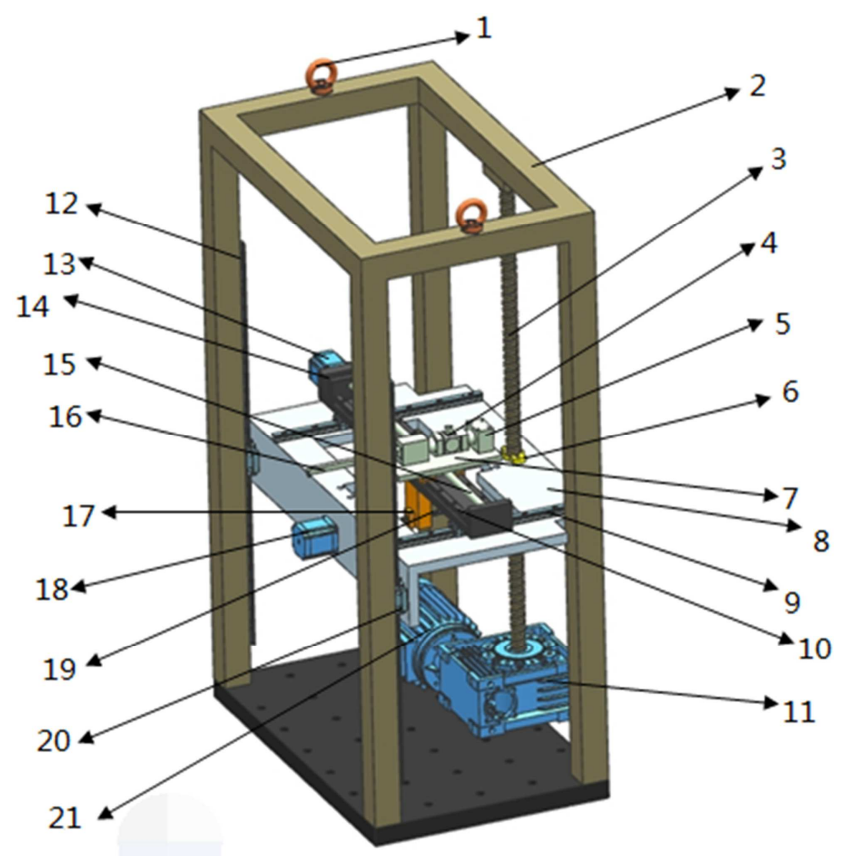

Figure 1. Three-dimensional sliding table structure design.

1. Ring; 2. Frame; 3. Screw 1; 4. Force sensor; 5. Fixed table; 6. Ball screw nut pair $1 ; 7$. Worktable; 8 . Tile table; 9 . Guide rail 1; 10. Guide rail 2; 11. Reducer; 12. Guide rail 3; 13. Motor 1; 14. Module; 15. Screw 2; 16. Manipulator; 17. Ball screw nut 2; 18. Motor 2; 19. Screw 3; 20. Slider; 21. Motor 3

Based on Dongling electromagnetic vibrating table and control device designed three-dimensional sliding shifting mechanism, and on the premise of ensuring reliability, the use of automatic positioning traction mechanism to avoid the random error caused by manual positioning and improve the detection efficiency And automation level. The key technology of the three-dimensional mobile station is the design of the platform body structure. Due to the limitation of the energy level of the vibration table, the dynamic and static contact of the circuit breaker must reach a critical state that will not break, and it is necessary to use a stretching mechanism to perform the position stretching operation to achieve this. [2] Working conditions. As shown in Figure. 1, the $\mathrm{X}$ - and Y-direction sliding stages are located on the
Z-direction sliding stage. In order to reduce the bearing weight of the screw 1, a set of latching devices is designed at the bottom of the Z-direction sliding stage, when the Z-direction sliding stage When the target height is reached, the bolt mechanism is extended and fixed on the wall of the electric control cabinet, which effectively reduces the friction and loss of the screw rod 1 under the vibration environment. It can be seen from Figure. 1 that the traction mechanism of the carrying tool table is composed of a Y-direction sliding stage and a hook, and the hook is used to connect with the movable contact of the load-carrying electrical device, and is driven through the Y-direction sliding stage to realize the movable contact. The purpose of traction. [3]

(2) Design of a tension mechanism for a low-voltage circuit breaker manipulator

In order to achieve the traction movement of the loader's electrical contact and the dynamic test of the low-voltage device, this technology proposes a molded case circuit breaker test platform system, which includes the design of the electrical control cabinet and the detection system, and ultimately realizes the mechanical arm. Three-dimensional movement in the three directions of X, Y, and Z. In the design of the electrical control cabinet system, a ball screw, a strain-type load cell, a ring screw, a stepper motor and a drive are selected. A controller of the Siemens CPU 224 XP CN is used to control the motor using a programming software.

In order to achieve the critical action of downloading the electrical contacts in vibration conditions, a mechanical arm positioning mechanism was designed on the basis of a three-dimensional sliding table, as shown in Figure. 1. According to the structural diagram of the three-dimensional sliding platform, the structure includes a load-bearing frame, a lifting ring, a worm gear worm lifter, a pedestal, a guide rail, a stepping motor, a slider, a workbench, and a robot arm. The robot arm is mounted on a workbench, as shown in the Figure. 2 shows that the robot arm is connected to the load cell and the load cell is connected to the fixed block. The fixed block is mounted on the workbench by bolt connection. The worktable is mounted on the slide block of the module, and the load cell and work are performed. Taiwan without contact. When the motor 1 is started, the screw rotates as the motor 1 rotates, and the table moves along the center line of the screw. [4] The stepper motor 3 is connected to the speed reducer to drive the screw rod 1 to rotate, and the screw rod 1 moves the pedestal up and down through the ball screw nut pair 1 so as to reach the up and down movement of the robot arm; at the same time, the motor 2 drives the rotation of the screw rod, The forward and backward movement of the module on the guide rail 1 is achieved by the ball screw nut pair 2, so that the robot arm moves back and forth; the rotation of the screw rod 2 is driven by the motor 1 to move the work table left and right on the module to realize the robot arm. Moves left and right so that the movement of the robot arm in three directions is achieved. $[5]$. 


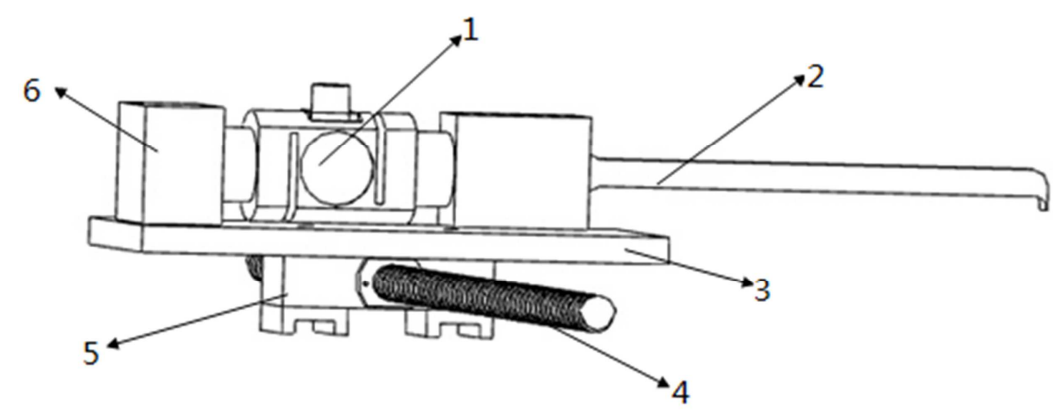

1. Weighing sensor 2. Robotic arm 3. Worktable 4. Screw 5. Slide 6. Fixed block

Figure 2. Arm positioning mechanism.

(3) Set up a test system for load-carrying appliances

According to the design requirements, the low-voltage circuit breaker vibration test system is developed based on the design results of the electric control cabinet system, including the selection of the PLC, [6] the motor and the driver. Selection, selection of eyebolts, control systems, etc. The design of the control system and detection system for the low voltage circuit breaker electrical control cabinet was completed.

The system control cabinet adopts PLC as the controller. Its design idea is shown in Figure. 3. The three-dimensional movement of the worktable $\mathrm{X}, \mathrm{Y}$ and $\mathrm{Z}$ can be achieved through the operation of the motor drive motor. In order to determine the dynamic characteristics of the load-carrying appliances, an analog vibration experiment was performed. An accelerometer was mounted on the vibratory table and another accelerometer was installed near the movable contact. As shown in Figure. 4, the 3-D mobile station was driven by PLC control. The robotic arm reaches the designated position and pulls the moving contact of the circuit breaker. At the same time, the magnitude of the pulling force is controlled by observing the "force \& strain comprehensive parameter tester" display force value, and then the contact is judged by observing the change of the voltage displayed by the "oscilloscope". The on and off, in order to determine the dynamic characteristics of the parameters. [7]

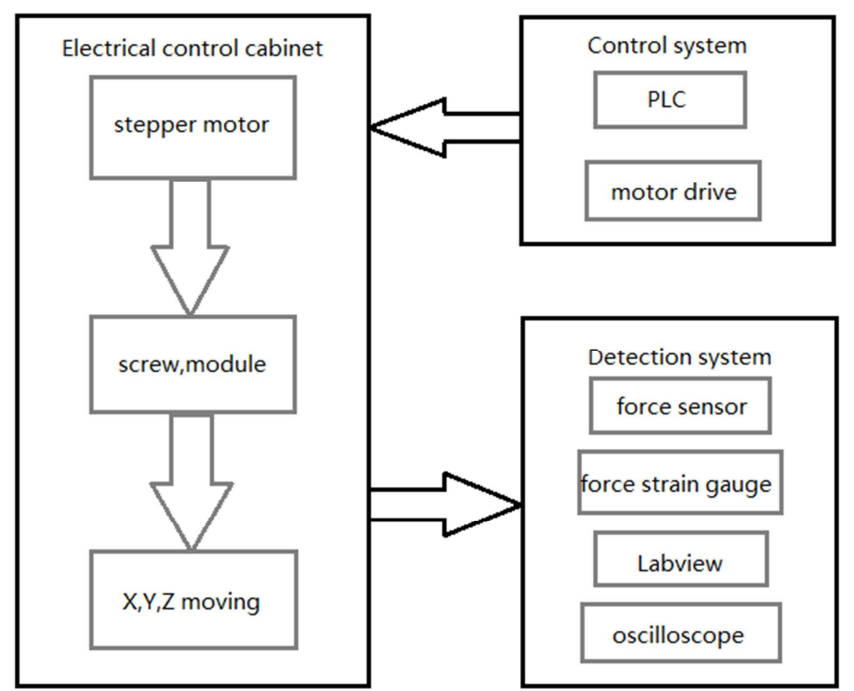

Figure 3. Design idea of Test platform system for carrying Electric Appliances.

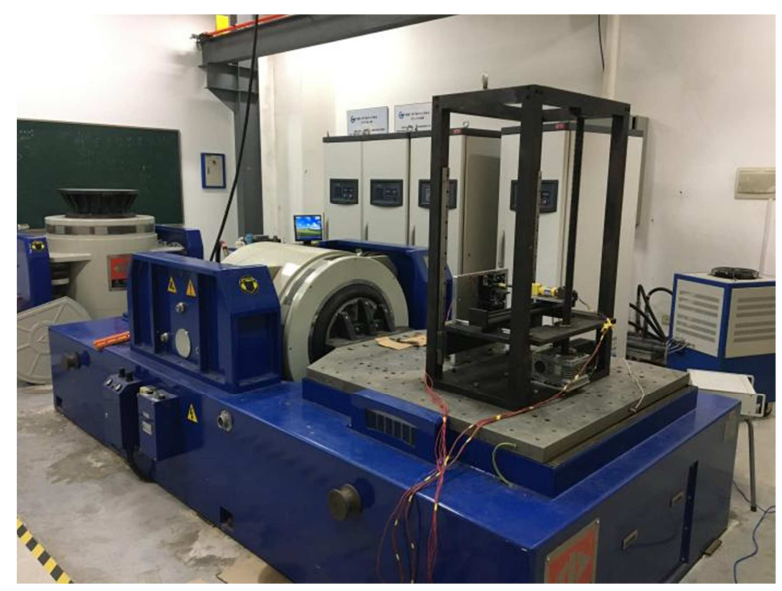

Figure 4. Vibration test system for low voltage electrical apparatus.

\section{Reliability Analysis of Platform Framework Based on ANSYS Finite Element Analysis}

\subsection{Finite Element Method Analysis}

(1) Introduction to Ansys Workbench

The modules in ANSYS Workbench not only contain most of the solving algorithms in ANSYS, but also a co-simulation platform for CAD and design processes. When the classic version of ANSYS is imported into the model, missing line, face, or body conditions often occur. However, ANSYS Workbench can seamlessly link with common CAD software, and feature deletions rarely occur. And in terms of contact, meshing, post-processing, etc., its operability is also more convenient, and the interface is more user-friendly.

(2) Automatic grid division

Meshing belongs to the pre-processing part of the finite element analysis. The general part will consume a lot of time. Workbench has the function of automatically dividing the grid, saving time, allowing researchers to put more effort into results analysis and product optimization. The meshing methods include Tetrahedrons, Sweep, MultiZone, Automaic, and Hex Dominant.

(3) Parametric design language

Parametric design language, English abbreviation APDL, it belongs to the ANSYS script tool, plays an important role in 
the ANSYS analysis. For example, parametric analysis of geometric models, analysis and extension of complex load boundary conditions and instrument processing results.

Workbench provides powerful application modules, mainly including:

Mechanical: The main structure analysis and thermodynamic analysis and dynamic analysis, which covers the meshing function. The

Mechanical APDL: The module uses a traditional operator interface to optimize the finite element analysis of advanced machinery.

Fluid Flow: Primarily for fluid dynamics.

Geometry: You can create the required model.

Engineering Data: Set the properties of the material. [8]

(4) Analysis steps of ANSYS Workbench

The analysis steps of ANSYS Workbench are shown in Figure 5. The general analysis steps include preliminary determination, pre-processing, solution and post-processing. Among them, the preliminary determination is mainly to determine the analysis type, unit type and model type; the pre-processing mainly includes establishing the model, importing the model, defining the material properties, and dividing the grid; the solution mainly includes setting and solving the load constraint; the main analysis result of the post-processing And verify.

\subsection{Platform Framework Modeling and Finite Element Analysis}

(1) Modal analysis of the platform framework

Before the modal analysis of the platform framework was performed, the framework was meshed using an automated division method. Clicking on Mesh resulted in 19689 nodes and 4029 units. Then Fixed Support is applied at the bottom of the frame to constrain the translation freedom in three directions on the ground. Extract the first six modes of the frame.

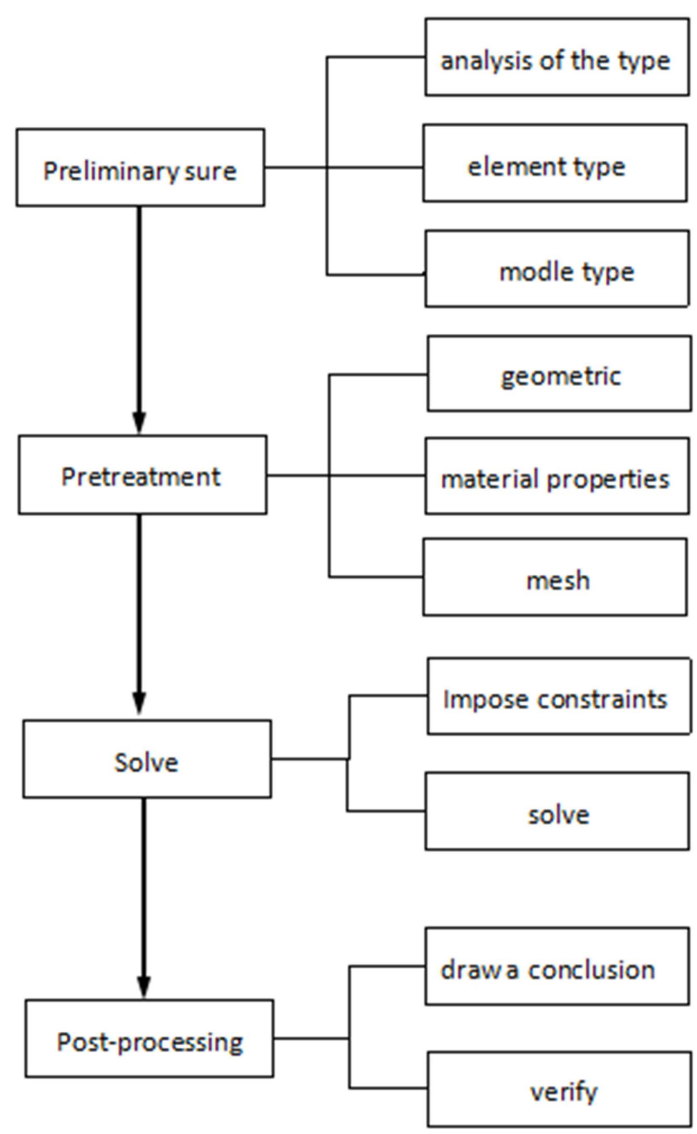

Figure 5. Analysis steps of ANSYS Workbench.

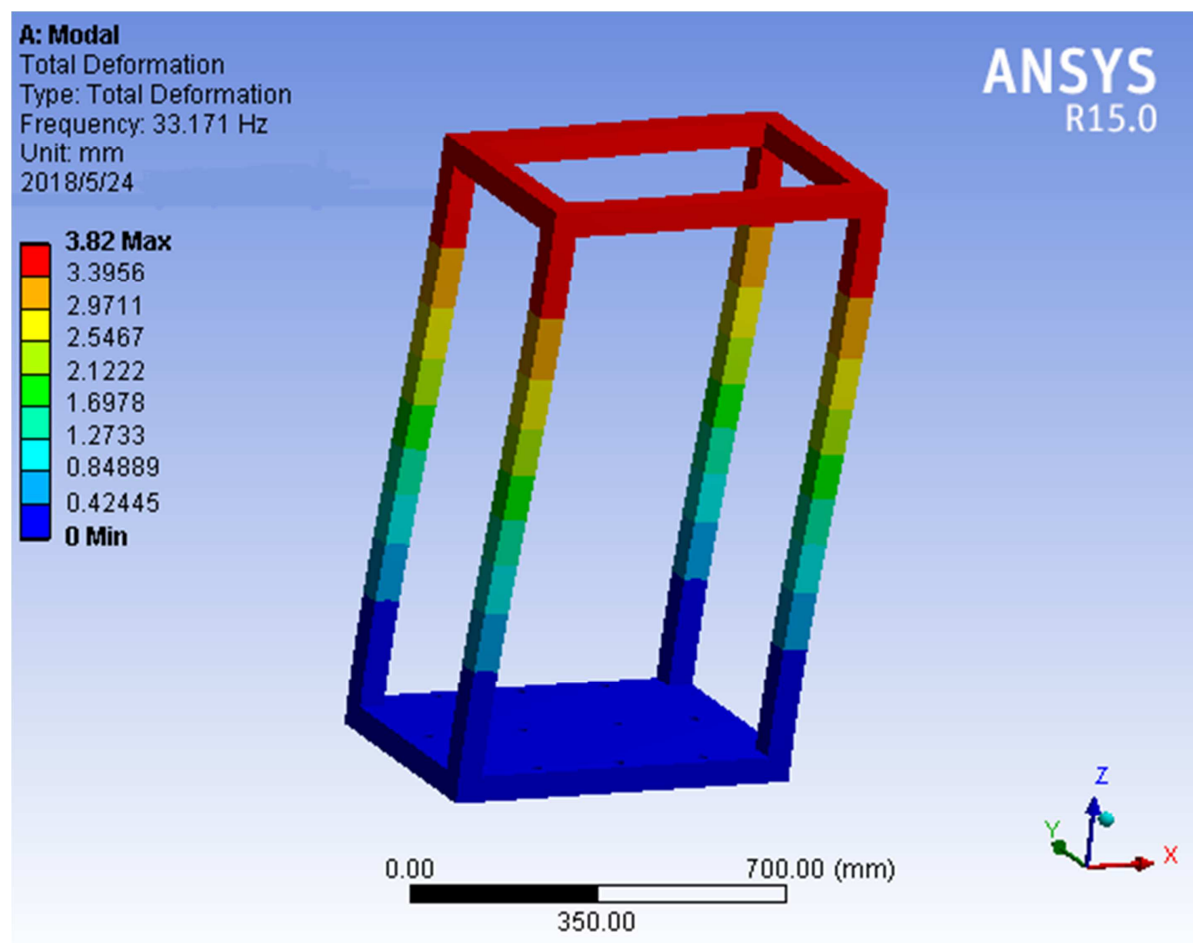

Figure 6. First-order modal mode. 


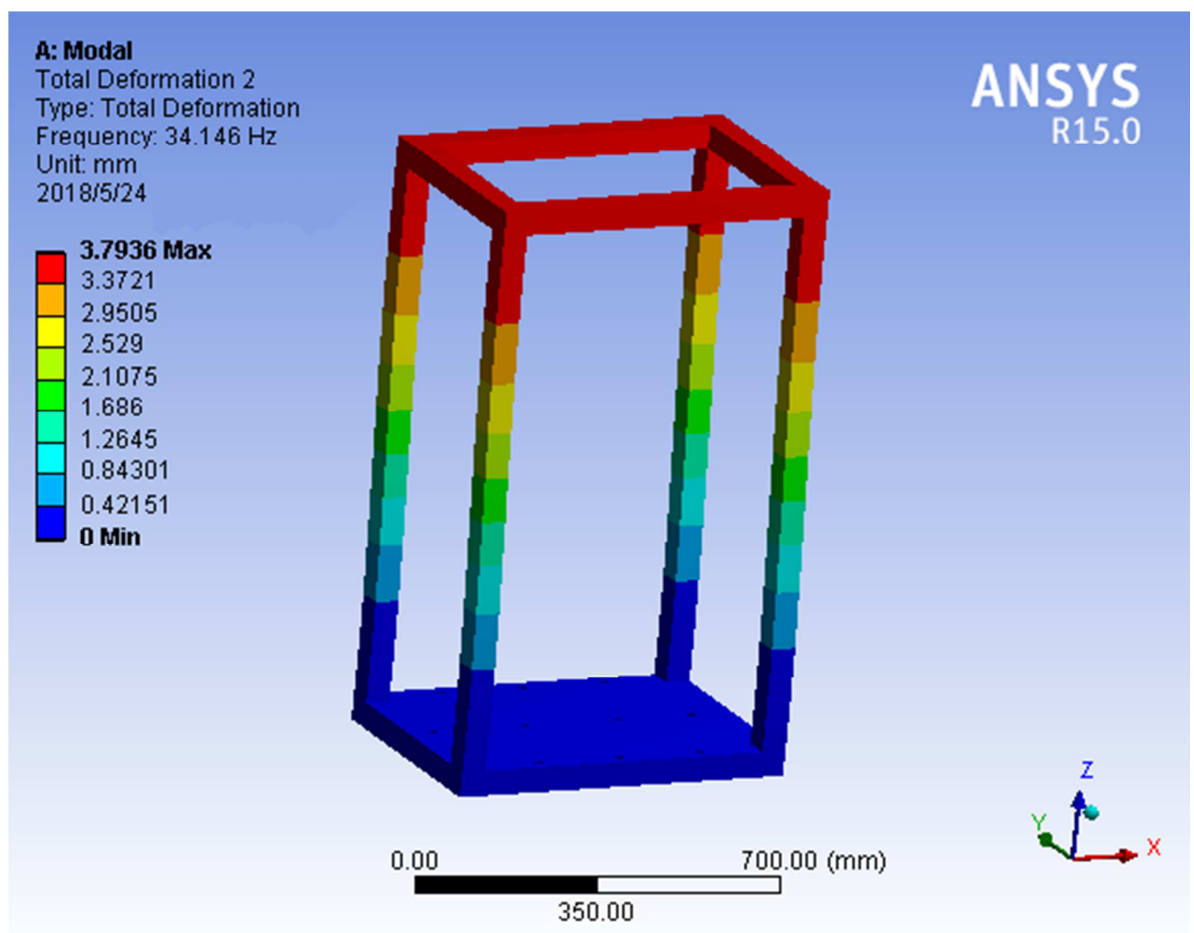

Figure 7. Second-order modal mode.

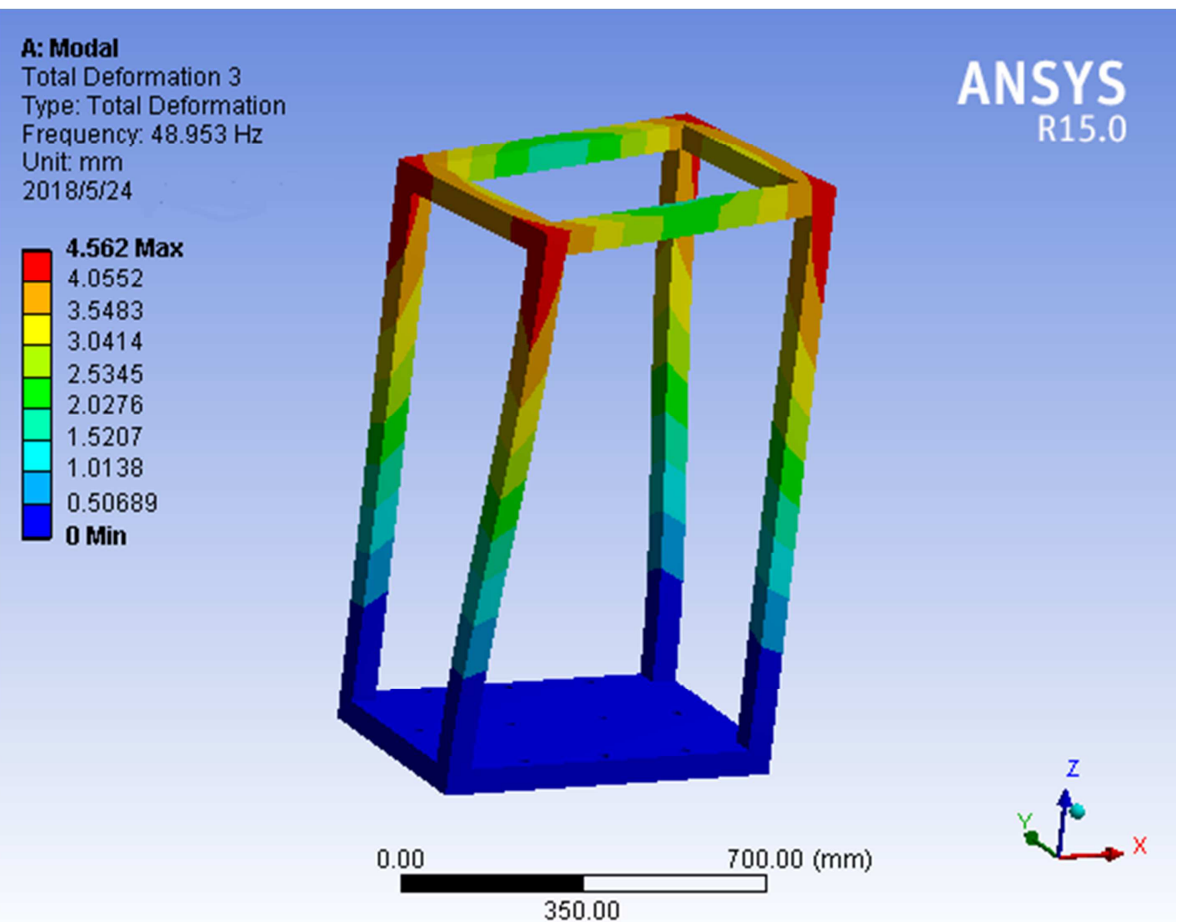

Figure 8. Third-order modal mode.

Table 1. Natural frequency of each mode.

\begin{tabular}{ll}
\hline Mode & Frequency/Hz \\
\hline 1 & 33.171 \\
2 & 34.146 \\
3 & 48.953 \\
4 & 160.97 \\
5 & 161.22 \\
6 & 174.75 \\
\hline
\end{tabular}




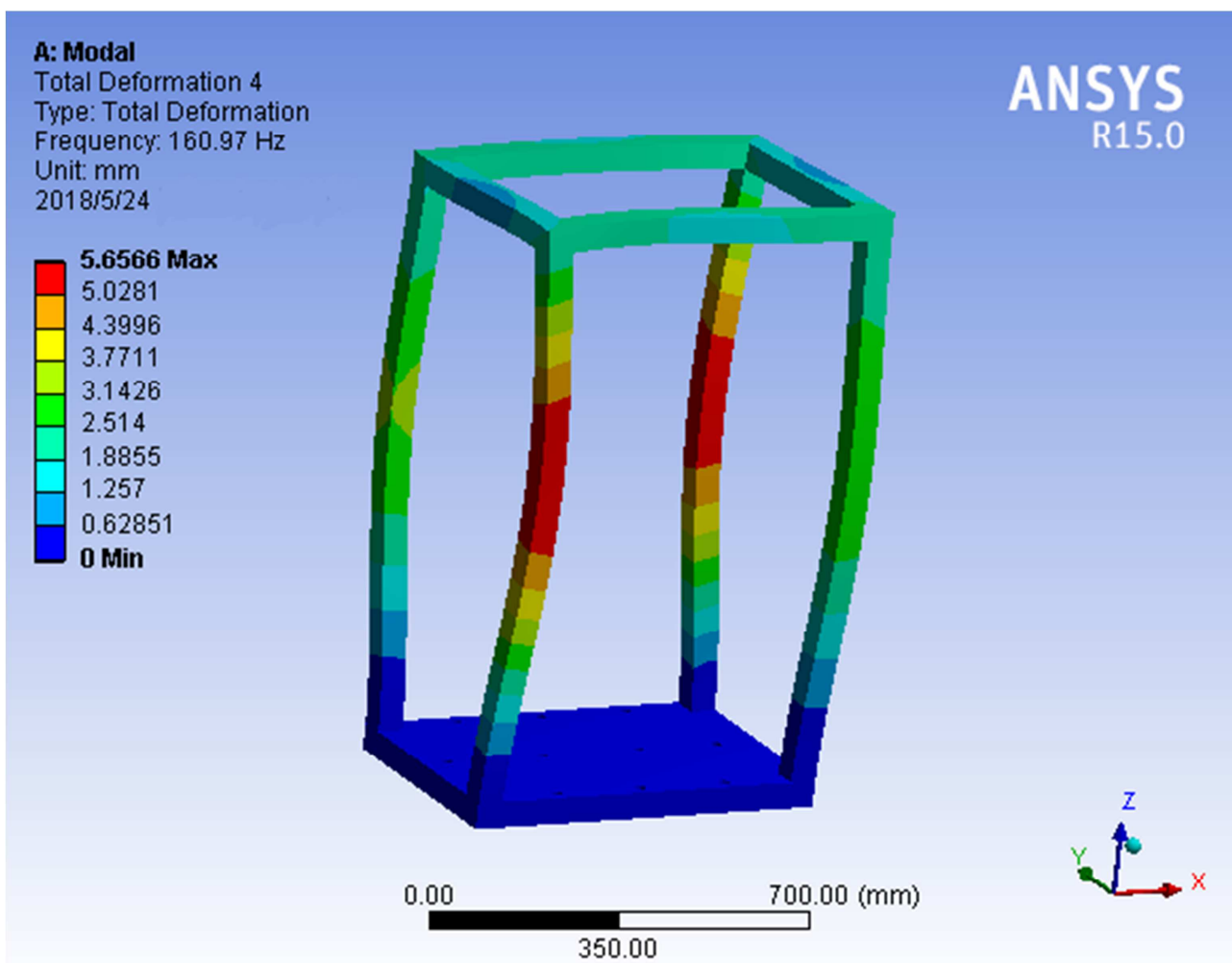

Figure 9. Fourth-order modal mode.

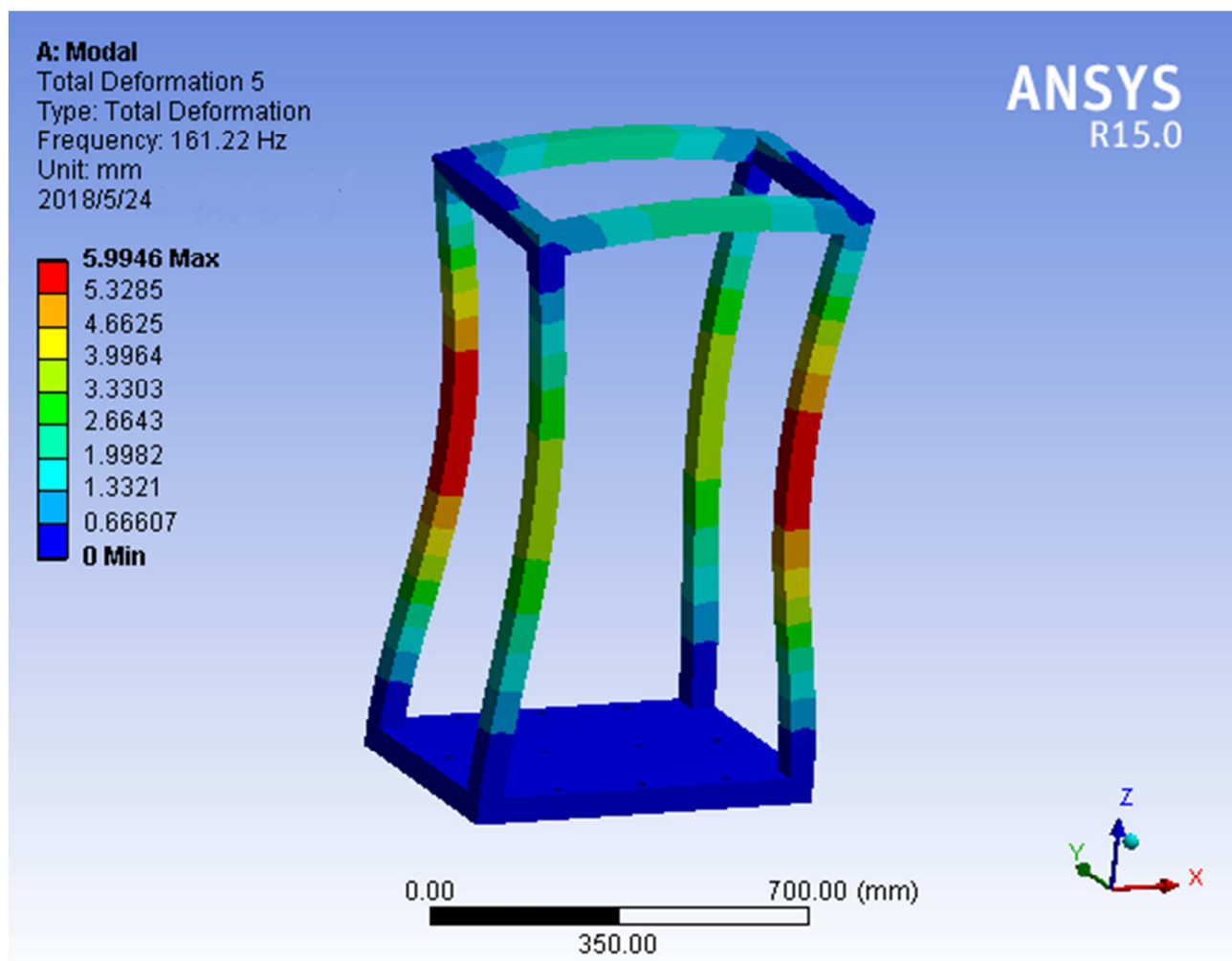

Figure 10. Fifth-order modal mode. 


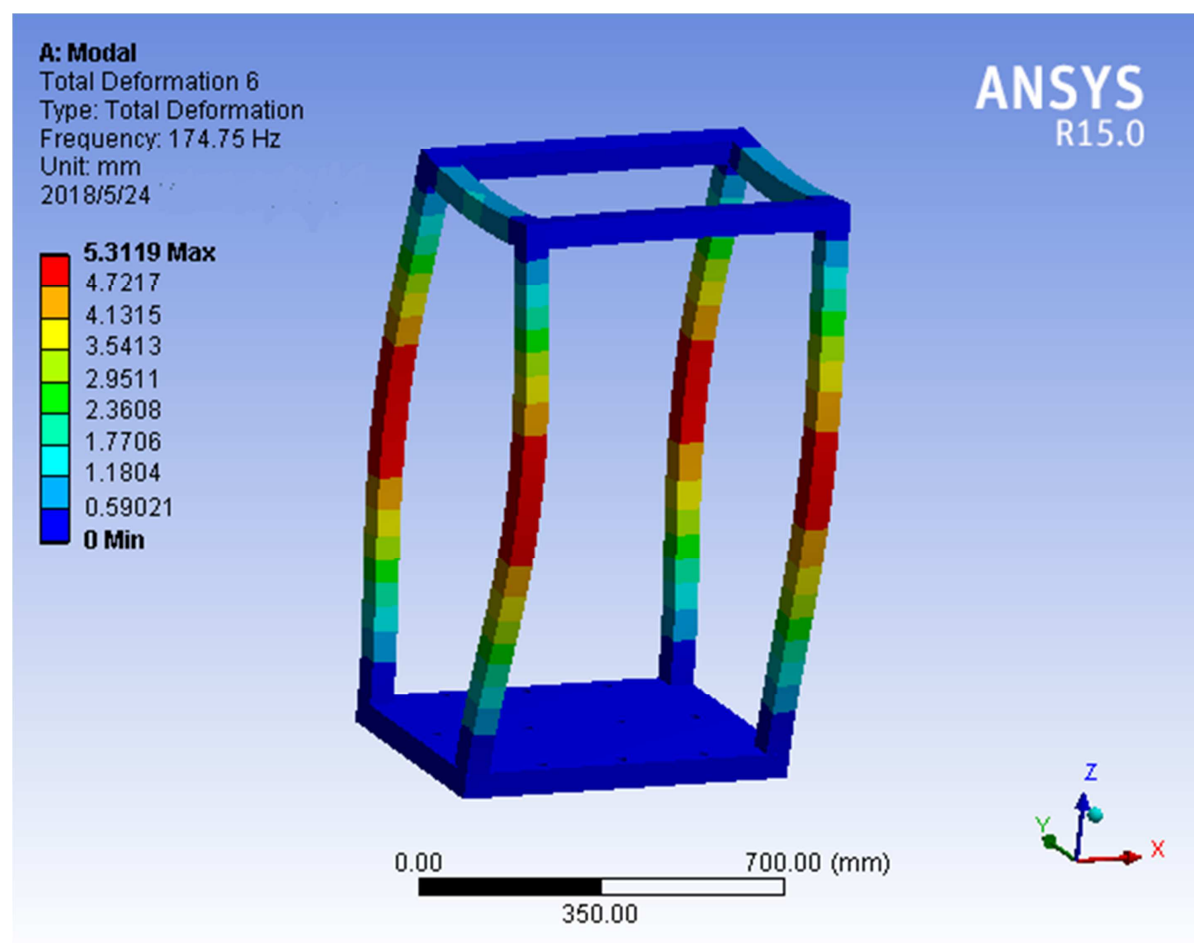

Figure 11. Sixth order modal mode.

(2) Analysis of results

According to the frame's various modes, the first three orders all occur in the XY plane. The maximum deformation occurs at the four top corners of the frame, and the natural frequencies are: $33.171 \mathrm{~Hz}, 34.146 \mathrm{~Hz}$, and $48.953 \mathrm{~Hz}$.. The 4 th, 5th and 6th orders are the bending deformation of the frame. The four columns are twisted. The maximum deformation occurs in the middle of the column and the natural frequencies are $160.97 \mathrm{~Hz}, 161.22 \mathrm{~Hz}$, and $174.75 \mathrm{~Hz}$, respectively.

\section{Conclusion}

In this paper, the design of the moulded case circuit breaker vibration test platform is firstly implemented modularly, and the main components and the designed structure are introduced. The feasibility of the platform construction is well explained. In addition, based on the analysis of the finite element, the reliability of the experimental framework of the platform was studied, and the deformation and natural frequency of the frame were analyzed. Finally, the results that meet the experimental requirements were obtained. The accuracy of the experiment and the safety of the equipment structure were also obtained. Sex provides a reference.

\section{References}

[1] Jixin, Wang Shurong. Adaptive design of vibration environment for electronic equipment $[\mathrm{M}]$. Beijing: Publishing House of Electronics Industry, 2012.
[2] Liu Ye. Analysis of Structural Design Method for Vibration System of Electrical Reliability Test Bench [J]. Science and Technology Guide, 2013, (26): 205.

[3] Jun Zhong, Wenyuan Li, Roy Billinton. Incorporating a condition monitoring based aging failure model of a circuit breaker in substation reliability assessment. [J]. IEEE Transactions on Power Systems, 2015, 30 (6):3407-3415.

[4] Zhong Guo-sheng, Ao Li-ping, Zhao Kui. Influence of explosion parameters on wavelet packet frequency band energy distribution of blast vibration. [J]. Journal of Central South University, 2012, (19):2674-2680.

[5] Lu Wei. Design of low-voltage electrical reliability test platform [D]. Shanghai: East China University of Science and Technology, 2016.

[6] Li He, Jiang Ning, Feng Jianqiang. Research on the closed electrical appliance in the low-voltage circuit breaker short-circuit test $[\mathrm{J}]$. Electric Apparatus and Energy Efficiency Management, 2017, (10):52-55.

[7] Han Junhua. Optimized design of hydraulic support base based on Pro/E and ANSYS Workbench [D]. Hebei University of Technology, 2016.

[8] Deng Lei. Research on the Teaching Reform of Electrical Control and PLC Applied Technology Course under Internet + Background [J]. China High-tech Zone, 2017, (24): 72.

[9] Ruan Hongyun. Research on AC servo motor drive and displacement control technology based on PLC [J]. Modern Manufacturing Technology and Equipment, 2017, (09):80-81.

[10] Zhang J F, M L Liu, et al. Mechanical Fault Diagnosis for HV Circuit Breakers Based on Ensemble Empirical Mode Decomposition Energy Entropy and Support Vector Machine. Mathematical Problems in Engineering, 2015, 101757(6pp). 